\title{
ON THE EXISTENCE OF LARGE ANTICHAINS FOR DEFINABLE QUASI-ORDERS
}

\author{
BENJAMIN D. MILLER AND ZOLTÁN VIDNYÁNSZKY
}

\begin{abstract}
We generalize Harrington-Marker-Shelah's Dilworthstyle characterization of the existence of non-empty perfect antichains to co-analytic quasi-orders, establish the analogous theorem at the next definable cardinal, and consider generalizations beyond the first level of the projective hierarchy.
\end{abstract}

\section{INTRODUCTION}

A quasi-order is a reflexive transitive binary relation. Associated with every such relation $R$ on a set $X$ are the equivalence relation $x \equiv_{R} y \Longleftrightarrow(x R y$ and $y R x)$ and the incomparability relation $x \perp_{R} y \Longleftrightarrow(\neg x R y$ and $\neg y R x)$. We say that a set $Y \subseteq X$ is an $R$-antichain if $R \uparrow Y$ is the diagonal on $Y$, and an $R$-chain if $\perp_{R}\lceil Y$ is empty. A subset of a topological space is perfect if it is closed and has no isolated points, Borel if it is in the $\sigma$-algebra generated by the open sets, analytic if it is a continuous image of a closed subset of $\mathbb{N}^{\mathbb{N}}$, and co-analytic if its complement is analytic. In $\$ 1$, we generalize [HMS88, Theorem 5.1] from Borel to co-analytic quasi-orders:

Theorem 1. Suppose that $X$ is a Hausdorff space and $R$ is a coanalytic quasi-order on $X$. Then exactly one of the following holds:

(1) The space $X$ is a union of countably-many Borel R-chains.

(2) There is a non-empty perfect $R$-antichain.

Our proof uses only Baire category arguments and the $\mathbb{G}_{0}$ dichotomy [KST99, Theorem 6.3], which itself has a classical proof [Mil12. An interesting new wrinkle is that, while such arguments typically utilize just one application of the $\mathbb{G}_{0}$ dichotomy, ours requires infinitely many.

2010 Mathematics Subject Classification. Primary 03E15, 28 A05.

Key words and phrases. Antichain, chain, dichotomy, Dilworth, HarringtonMarker-Shelah, perfect, quasi-order, smooth.

Both authors were supported in part by FWF Grants P28153 and P29999, and the second in part by the National Research, Development, and Innovation Office - NKFIH Grants 113047, 104178, and 124749. 
A homomorphism from a binary relation $R$ on $X$ to a binary relation $S$ on $Y$ is a function $\phi: X \rightarrow Y$ such that $(\phi \times \phi)(R) \subseteq S$, and a reduction of $R$ to $S$ is a homomorphism from $R$ to $S$ that is also a homomorphism from $\sim R$ to $\sim S$. A Borel equivalence relation $E$ on an analytic Hausdorff space $X$ is smooth if there is a Borel-measurable reduction of $E$ to equality on $2^{\mathbb{N}}$, and an analytic set $A \subseteq X$ is $E$ smooth if $E \uparrow A$ is smooth. In \$2, we establish the analog of [HMS88, Theorem 5.1] at the next Borel cardinal:

Theorem 2. Suppose that $X$ is an analytic Hausdorff space and $R$ is a Borel quasi-order on $X$. Then exactly one of the following holds:

(1) There is a smooth Borel superequivalence relation of $\equiv_{R}$ whose equivalence classes are $R$-chains.

(2) There is an $\equiv_{R}$-non-smooth perfect set whose quotient by $\equiv_{R}$ is an $\left(R / \equiv_{R}\right)$-antichain.

Our proof uses only Baire category arguments and the $\mathbb{E}_{0}$ dichotomy [HKL90, Theorem 1.1], which itself has a classical proof [Mil12], and reveals that the theorem holds for the rather degenerate reason that its two alternatives are equivalent to those of the $\mathbb{E}_{0}$ dichotomy (for $\equiv_{R}$ ).

A subset of a topological space $X$ is $\kappa$-Borel if it is in the $\kappa$-complete algebra generated by the open sets, $\kappa$-Souslin if it is a continuous image of a closed subset of $\kappa^{\mathbb{N}}$, co- $\kappa$-Souslin if its complement is $\kappa$-Souslin, $b i$ - $\kappa$-Souslin if it is both $\kappa$-Souslin and co- $\kappa$-Souslin, and $\aleph_{0}$-universally Baire if its pre-image under every continuous function $\phi: 2^{\mathbb{N}} \rightarrow X$ has the Baire property. Let $\mathbb{E}_{0}$ denote the equivalence relation on $2^{\mathbb{N}}$ given by $c \mathbb{E}_{0} d \Longleftrightarrow \exists n \in \mathbb{N} \forall m \geq n c(m)=d(m)$. An embedding is an injective reduction. In $\oint 3$, we note that our arguments also yield:

Theorem 3. Suppose that $\kappa$ is an aleph, $X$ is a Hausdorff space, and $R$ is an $\aleph_{0}$-universally-Baire co- $\kappa$-Souslin quasi-order on $X$. Then at least one of the following holds:

(1) The space $X$ is a union of $\kappa$-many $R$-chains.

(2) There is a non-empty perfect $R$-antichain.

Theorem 4. Suppose that $\kappa$ is an aleph, $X$ is a Hausdorff space, and $R$ is an $\aleph_{0}$-universally-Baire bi- $\kappa$-Souslin quasi-order on $X$. Then at least one of the following holds:

(1) There is a homomorphism from $\equiv_{R}$ to equality on $2^{\kappa}$ such that the pre-image of every singleton is an $R$-chain.

(2) There is a continuous embedding $\pi: 2^{\mathbb{N}} \rightarrow X$ of $\mathbb{E}_{0}$ into $\equiv_{R}$ such that $\pi\left(2^{\mathbb{N}}\right) / \equiv_{R}$ is an $\left(R / \equiv_{R}\right)$-antichain. 
In the special case that $X$ is analytic, one can use the (non-classical) arguments of [Kan97] to establish the strengthenings in which the objects in condition (1) of these results are $\kappa^{+}$-Borel $/ \kappa^{+}$-Borel measurable.

A subset of an analytic Hausdorff space is $\boldsymbol{\Sigma}_{1}^{1}$ if it is analytic, $\boldsymbol{\Pi}_{n}^{1}$ if its complement is $\boldsymbol{\Sigma}_{n}^{1}, \boldsymbol{\Sigma}_{n+1}^{1}$ if it is a continuous image of a $\boldsymbol{\Pi}_{n}^{1}$ set, and $\boldsymbol{\Delta}_{n}^{1}$ if it is both $\boldsymbol{\Pi}_{n}^{1}$ and $\boldsymbol{\Sigma}_{n}^{1}$. Let $\boldsymbol{\delta}_{n}^{1}$ denote the supremum of the lengths of all $\boldsymbol{\Delta}_{n}^{1}$ pre-wellorderings of $\mathbb{N}^{\mathbb{N}}$. We say that a $\boldsymbol{\Delta}_{2 n+1}^{1}$ equivalence relation $E$ on an analytic Hausdorff space $X$ is smooth if there exists $\kappa<\boldsymbol{\delta}_{2 n+1}^{1}$ for which there is a $\boldsymbol{\Delta}_{2 n+1}^{1}$-measurable reduction of $E$ to equality on $2^{\kappa}$, and an analytic set $A \subseteq X$ is E-smooth if $E \uparrow A$ is smooth. Taking the known structure theory of the projective sets as a black box, we note that our arguments also provide classical proofs of the relevant special cases of the Kanovei-style strengthenings of Theorems 3 and 4 necessary to obtain:

Theorem $5(\mathrm{AD})$. Suppose that $n \in \mathbb{N}, X$ is an analytic Hausdorff space, and $R$ is a $\Pi_{2 n+1}^{1}$ quasi-order on $X$. Then exactly one of the following holds:

(1) The space $X$ is a union of $\left(<\boldsymbol{\delta}_{2 n+1}^{1}\right)$-many $\Delta_{2 n+1}^{1} R$-chains.

(2) There is a non-empty perfect $R$-antichain.

Theorem 6 (AD). Suppose that $n \in \mathbb{N}, X$ is an analytic Hausdorff space, and $R$ is a $\boldsymbol{\Delta}_{2 n+1}^{1}$ quasi-order on $X$. Then exactly one of the following holds:

(1) There is a smooth $\Delta_{2 n+1}^{1}$ superequivalence relation of $\equiv_{R}$ whose equivalence classes are $R$-chains.

(2) There is an $\equiv_{R}$-non-smooth perfect set whose quotient by $\equiv_{R}$ is an $\left(R / \equiv_{R}\right)$-antichain.

In a future paper with Müller, we will establish the version of the $\mathbb{G}_{0}$ dichotomy necessary to obtain such results from PD.

We work in ZF + DC throughout.

\section{Perfect antichains}

For each discrete set $D$ and sequence $s \in D^{<\mathbb{N}}$, we use $\mathcal{N}_{s}$ to denote the basic open set consisting of all extensions of $s$ in $D^{\mathbb{N}}$. We use the notation $\forall^{*} x \in X P(x)$ to indicate that $\{x \in X \mid \neg P(x)\}$ is meager, and $\exists^{*} x \in X P(x)$ to indicate that $\{x \in X \mid P(x)\}$ is non-meager. Fix sequences $s_{n} \in 2^{n}$ such that $\forall s \in 2^{<\mathbb{N}} \exists n \in \mathbb{N} s \sqsubseteq s_{n}$, and define $\mathbb{G}_{0}=\bigcup_{n \in \mathbb{N}}\left\{\left(\mathbb{S}_{n} \frown(i) \frown c, \mathbb{S}_{n} \frown(1-i) \frown c\right) \mid c \in 2^{\mathbb{N}}\right.$ and $\left.i<2\right\}$. While our proof of the characterization of the existence of a non-empty perfect antichain requires infinitely-many applications of the $\mathbb{G}_{0}$ dichotomy, we need only one to establish the following: 
Theorem 7. Suppose that $X$ is a Hausdorff space, $R$ is a co-analytic quasi-order on $X$, and $X$ is not a union of countably-many Borel $R$ chains. Then there are compact sets $K_{i} \subseteq X$ that are not unions of countably-many Borel $R$-chains such that $\prod_{i<2} K_{i} \subseteq \perp_{R}$.

Proof. As $\perp_{R}$ is analytic and $X$ is not a union of countably-many Borel $R$-chains, the $\mathbb{G}_{0}$ dichotomy yields a continuous homomorphism $\phi: 2^{\mathbb{N}} \rightarrow X$ from $\mathbb{G}_{0}$ to $\perp_{R}$. As the set $R_{0}=(\phi \times \phi)^{-1}(R)$ is co-analytic, it has the Baire property (see, for example, [Kec95, 21.6]), thus so too does $\perp_{R_{0}}$.

Lemma 8. The relation $\perp_{R_{0}}$ is non-meager.

Proof. Suppose, towards a contradiction, that $\perp_{R_{0}}$ is meager, and fix non-empty open sets $U_{i} \subseteq 2^{\mathbb{N}}$ for which $R_{0}$ is comeager in $\prod_{i<2} U_{i}$ (see, for example, [Kec95, Proposition 8.26]). The Kuratowski-Ulam theorem (see, for example, Kec95, Theorem 8.41]) ensures that the sets $C_{0}=\left\{c \in 2^{\mathbb{N}} \mid \exists^{*} d \in U_{0} c R_{0} d\right\}$ and $C_{1}=\left\{d \in 2^{\mathbb{N}} \mid \forall^{*} c \in U_{0} c R_{0} d\right\}$ have comeager union, and [Kec95, Theorem 16.1] and the KuratowskiUlam theorem imply that they have the Baire property. The Kuratowski-Ulam theorem also ensures that $C_{0}$ is non-meager, since otherwise $\forall^{*} c, d \in U_{0}\left(\neg c R_{0} d\right.$ and $\left.d R_{0} c\right)$, and $C_{1}$ is non-meager. As the $\mathbb{E}_{0^{-}}$ saturation of every non-meager set with the Baire property is comeager (see, for example, [Kec95, Theorem 8.47]), there are comeagerly-many $c \in 2^{\mathbb{N}}$ for which the sets $C_{i} \cap[c]_{\mathbb{E}_{0}}$ non-trivially partition $[c]_{\mathbb{E}_{0}}$. As a straightforward induction reveals that $\mathbb{E}_{0}$ is the equivalence relation generated by $\mathbb{G}_{0}$, it follows that $\left(\prod_{i<2} C_{i}\right) \cap \mathbb{G}_{0} \neq \emptyset$, contradicting the fact that $\prod_{i<2} C_{i} \subseteq R_{0}$.

Lemma 9. There are continuous homomorphisms $\phi_{i}: 2^{\mathbb{N}} \rightarrow 2^{\mathbb{N}}$ from $\mathbb{G}_{0}$ to itself for which $\prod_{i<2} \phi_{i}\left(2^{\mathbb{N}}\right) \subseteq \perp_{R_{0}}$.

Proof. By Lemma 8, there are non-empty open sets $U_{i} \subseteq 2^{\mathbb{N}}$ and dense open sets $V_{n} \subseteq \prod_{i<2} U_{i}$ such that $\bigcap_{n \in \mathbb{N}} V_{n} \subseteq \perp_{R_{0}}$. Recursively construct $u_{i, n} \in 2^{<\mathbb{N}}$ and $k_{i, n} \in \mathbb{N}$ such that $\prod_{i<2} \mathcal{N}_{\phi_{i, n}\left(t_{i}\right)} \subseteq V_{n}$ for all $t_{0}, t_{1} \in 2^{n}$ and $\phi_{i, n}\left(s_{n}\right)=s_{k_{i, n}}$ for all $i<2$, where $\phi_{i, n}: 2^{n} \rightarrow 2^{<\mathbb{N}}$ is given by $\phi_{i, n}(t)=u_{i, 0} \frown \bigoplus_{m<n}(t(m)) \frown u_{i, m+1}$. Then the functions $\phi_{i}: 2^{\mathbb{N}} \rightarrow 2^{\mathbb{N}}$ given by $\phi_{i}(c)=\bigcup_{n \in \mathbb{N}} \phi_{i, n}(c \nmid n)$ are as desired.

It only remains to observe that if the functions $\phi_{i}$ are as in Lemma 9, then the sets $K_{i}=\left(\phi \circ \phi_{i}\right)\left(2^{\mathbb{N}}\right)$ are as desired.

We now establish our characterization of the existence of a nonempty perfect antichain: 
Proof of Theorem 1. Conditions (1) and (2) are clearly mutually exclusive. To see $\neg(1) \Longrightarrow(2)$, note that if condition (1) fails, then $X$ is the projection onto either coordinate of the complement of $\equiv_{R}$, thus analytic. Fix a continuous surjection $\phi: \mathbb{N}^{\mathbb{N}} \rightarrow X$, and recursively appeal to Theorem 7 to obtain functions $\psi_{n}: 2^{n} \rightarrow \mathbb{N}^{n}$ and sequences $\left(F_{s}\right)_{s \in 2^{n}}$ of closed subsets of $X$ with the following properties:

(1) $\forall s \in 2^{n} F_{s}$ is not a union of countably-many Borel $R$-antichains.

(2) $\forall s \in 2^{n} F_{s} \subseteq \phi\left(\mathcal{N}_{\psi_{n}(s)}\right)$.

(3) $\forall s \in 2^{n} F_{s \frown(0)} \cup F_{s \wedge(1)} \subseteq F_{s}$.

(4) $\forall s \in 2^{n} F_{s \frown(0)} \times F_{s \frown(1)} \subseteq \perp_{R}$.

(5) $\forall i<2 \forall s \in 2^{n} \psi_{n}(s) \sqsubseteq \psi_{n+1}(s \frown(i))$.

Define $\psi: 2^{\mathbb{N}} \rightarrow \mathbb{N}^{\mathbb{N}}$ by $\psi(c)=\bigcup_{n \in \mathbb{N}} \psi_{n}(c\lceil n)$, as well as $\pi=\phi \circ \psi$, noting that $\pi(c) \in \bigcap_{n \in \mathbb{N}} F_{c \mid n}$ for all $c \in 2^{\mathbb{N}}$. To see that $\pi\left(2^{\mathbb{N}}\right)$ is a perfect $R$-antichain, observe that if $c, d \in 2^{\mathbb{N}}$ are distinct, then there is a maximal natural number $n \in \mathbb{N}$ for which $c \uparrow n=d \uparrow n$, so the fact that $\pi(c) \in F_{s \frown(c(n))}$ and $\pi(d) \in F_{s \frown(d(n))}$ ensures that $\pi(c) \perp_{R} \pi(d)$. $\quad \nabla$

\section{Non-SMOOTH ANTICHAINS}

We now establish our characterization of the existence of a nonsmooth perfect set whose quotient is an antichain:

Proof of Theorem 2. To see that conditions (1) and (2) are mutually exclusive, note that if $E$ is a Borel superequivalence relation of $\equiv_{R}$ whose classes are $R$-chains, and $A \subseteq X$ is a set whose quotient by $\equiv_{R}$ is an $\left(R / \equiv_{R}\right)$-antichain, then $\equiv_{R} \uparrow A=E \uparrow A$. When $A$ is analytic, it follows that if $E$ is smooth, then so too is $\equiv_{R} \uparrow A$.

To see $\neg(1) \Longrightarrow(2)$, note that if (1) fails, then $\equiv_{R}$ is non-smooth, so the $\mathbb{E}_{0}$ dichotomy yields a continuous embedding $\phi: 2^{\mathbb{N}} \rightarrow X$ of $\mathbb{E}_{0}$ into $\equiv_{R}$. As the set $R_{0}=(\phi \times \phi)^{-1}(R)$ is Borel, it has the Baire property, thus so too does $\perp_{R_{0}}$.

Lemma 10. The relation $\perp_{R_{0}}$ is comeager.

Proof. If there exist $n \in \mathbb{N}$ and $s, t \in 2^{n}$ for which $R_{0}$ is comeager in $\mathcal{N}_{s} \times \mathcal{N}_{t}$, then the fact that $\mathbb{E}_{0} \subseteq R_{0}$ ensures that $R_{0}$ is comeager in $\mathcal{N}_{s^{\prime}} \times \mathcal{N}_{t^{\prime}}$ for all $s^{\prime}, t^{\prime} \in 2^{n}$, and therefore comeager, thus so too is $\equiv_{R_{0}}$, contradicting the fact that the latter set is $\mathbb{E}_{0}$.

Lemma 11. There is a continuous embedding $\psi: 2^{\mathbb{N}} \rightarrow 2^{\mathbb{N}}$ of $\mathbb{E}_{0}$ into itself that is also a homomorphism from $\sim \mathbb{E}_{0}$ to $\perp_{R_{0}}$.

Proof. By Lemma 10, there are dense open sets $U_{n} \subseteq 2^{\mathbb{N}} \times 2^{\mathbb{N}}$ such that $\bigcap_{n \in \mathbb{N}} U_{n} \subseteq \perp_{R_{0}}$. We can clearly assume that these sets are decreasing and disjoint from the diagonal. Recursively construct $u_{i, n} \in 2^{<\mathbb{N}}$ such 
that $\left|u_{0, n}\right|=\left|u_{1, n}\right|$ and $\prod_{i<2} \mathcal{N}_{\psi_{n+1}\left(t_{i} \frown(i)\right)} \subseteq U_{n}$ for all $t_{0}, t_{1} \in 2^{n}$, where $\psi_{n+1}: 2^{n+1} \rightarrow 2^{<\mathbb{N}}$ is given by $\psi_{n+1}(t)=\bigoplus_{m \leq n}(t(m)) \frown u_{t(m), m}$. Then the map $\psi: 2^{\mathbb{N}} \rightarrow 2^{\mathbb{N}}$ given by $\psi(c)=\bigcup_{n \in \mathbb{N}} \psi_{n}(c\lceil n)$ is as desired.

It only remains to observe that if the function $\psi$ is as in Lemma 11, then the set $(\phi \circ \psi)\left(2^{\mathbb{N}}\right)$ is as desired.

\section{Generalizations}

Simplifications of the classical proofs of the $\mathbb{G}_{0}$ and $\mathbb{E}_{0}$ dichotomies can be used to obtain a continuous homomorphism from $\mathbb{G}_{0}$ to every $\kappa$ Souslin graph on a Hausdorff space with no $\kappa$-coloring, and a continuous embedding of $\mathbb{E}_{0}$ into every bi- $\kappa$-Souslin equivalence relation on a Hausdorff space which is not reducible to equality on $2^{\kappa}$ [Mil12]. By using these facts in lieu of the usual dichotomies in our proofs of Theorems 1 and 2, we obtain proofs of Theorems 3 and 4 .

If $\mathrm{AD}$ holds and $n \in \mathbb{N}$, then a subset of an analytic Hausdorff space is $\boldsymbol{\Delta}_{2 n+1}^{1}$ if and only if it is $\boldsymbol{\delta}_{2 n+1}^{1}$-Borel [Mar70, Mos71], and there is a cardinal $\boldsymbol{\kappa}_{2 n+1}^{1}$ for which $\boldsymbol{\delta}_{2 n+1}^{1}=\left(\boldsymbol{\kappa}_{2 n+1}^{1}\right)^{+}$[Kec74] and a subset of an analytic Hausdorff space is $\boldsymbol{\Sigma}_{2 n+1}^{1}$ if and only if it is $\boldsymbol{\kappa}_{2 n+1}^{1}$-Souslin (see, for example, JJac08, Theorem 2.21]). It follows that continuous

images of $\boldsymbol{\delta}_{2 n+1}^{1}$-Borel sets are $\boldsymbol{\kappa}_{2 n+1}^{1}$-Souslin, a fact which alone ensures that the classical proofs of the $\mathbb{G}_{0}$ and $\mathbb{E}_{0}$ dichotomies yield the special cases of the Kanovei-style generalizations thereof at $\boldsymbol{\kappa}_{2 n+1}^{1}$. By using these in lieu of the usual dichotomies in our proofs of Theorems 1 and 2. we obtain proofs of the Kanovei-style strengthenings of Theorems 3 and 4 at $\boldsymbol{\kappa}_{2 n+1}^{1}$. As AD also ensures that every subset of a topological space is $\aleph_{0}$-universally Baire (see, for example, Kec95, Theorem 38.17]), Theorems 5 and 6 easily follow.

Acknowledgements. We would like to thank Sandra Müller for a useful conversation concerning the results of $\$ 3$.

\section{REFERENCES}

[HKL90] L. A. Harrington, A. S. Kechris, and A. Louveau, A Glimm-Effros dichotomy for Borel equivalence relations, J. Amer. Math. Soc. 3 (1990), no. 4, 903-928. MR 1057041

[HMS88] L. A. Harrington, D. Marker, and S. Shelah, Borel orderings, Trans. Amer. Math. Soc. 310 (1988), no. 1, 293-302. MR 965754

[Jac08] S. Jackson, Suslin cardinals, partition properties, homogeneity. Introduction to Part II, Games, scales, and Suslin cardinals. The Cabal Seminar. Vol. I, Lect. Notes Log., vol. 31, Assoc. Symbol. Logic, Chicago, IL, 2008, pp. 273-313. MR 2463617 
[Kan97] V. Kanovei, Two dichotomy theorems on colourability of non-analytic graphs, Fund. Math. 154 (1997), no. 2, 183-201, European Summer Meeting of the Association for Symbolic Logic (Haifa, 1995). MR 1477757 (98m:03103)

[Kec74] A. S. Kechris, On projective ordinals, J. Symbolic Logic 39 (1974), 269282. MR 0398833

[Kec95] _ Classical descriptive set theory, Graduate Texts in Mathematics, vol. 156, Springer-Verlag, New York, 1995. MR 1321597 (96e:03057)

[KST99] A. S. Kechris, S. Solecki, and S. Todorcevic, Borel chromatic numbers, Adv. Math. 141 (1999), no. 1, 1-44. MR 1667145

[Mar70] D. A. Martin, Pleasant and unpleasant consequences of determinateness, unpublished manuscript, March 1970.

[Mil12] B. D. Miller, The graph-theoretic approach to descriptive set theory, Bull. Symbolic Logic 18 (2012), no. 4, 554-575. MR 3053069

[Mos71] Y. N. Moschovakis, Uniformization in a playful universe, Bull. Amer. Math. Soc. 77 (1971), 731-736. MR 0285390

Benjamin D. Miller, Kurt Gödel Research Center for MathematiCal Logic, Universität Wien, Währinger Strasse 25, 1090 Wien, AusTRIA

E-mail address: benjamin.miller@univie.ac.at

$U R L$ : http://www.logic.univie.ac.at/benjamin.miller

Zoltán Vidnyánszky, Kurt Gödel Research Center for Mathematical Logic, Universität Wien, Währinger Strasse 25, 1090 Wien, AusTRIA

E-mail address: zoltan.vidnyanszky@univie.ac.at

$U R L$ : http://www.logic.univie.ac.at/ vidnyanszz77/ 